\title{
Evaluation of Health Care Professional Response To Patient-Reported Distress In Gynecologic Oncology Clinics At An Academic Cancer Centre
}

\section{Soha Atallah ( $\square$ soha_atallah@hotmail.com )}

Princess Margaret Hospital Cancer Centre https://orcid.org/0000-0001-6802-6790 Jackie Bender

University of Toronto Dalla Lana School of Public Health

\section{Anthony Fyles}

Princess Margaret Hospital Cancer Centre

\section{Kathy Han}

Princess Margaret Hospital: Princess Margaret Hospital Cancer Centre

\section{Michael Milosevic}

Princess Margaret Hospital Cancer Centre

\section{Sarah E. Ferguson}

Princess Margaret Hospital Cancer Centre

\section{Stephanie L'heureux}

Princess Margaret Hospital Cancer Centre

\section{ZhihuiAmy Liu}

University of Toronto Dalla Lana School of Public Health

Jennifer Croke

Princess Margaret Hospital Cancer Centre

\section{Research Article}

Keywords: Patient reported outcomes, oncology, distress

Posted Date: August 30th, 2021

DOl: https://doi.org/10.21203/rs.3.rs-802347/v1

License: (c) (i) This work is licensed under a Creative Commons Attribution 4.0 International License. Read Full License 


\section{Abstract}

Objective: To evaluate health care professional (HCP) documentation of elevated patient-reported symptoms and subsequent intervention in gynecologic oncology clinics.

Methods: This was a retrospective chart review of gynecologic oncology patients within a single institution. Prior to their clinical encounter, patients complete the validated Edmonton Symptom Assessment System revised (ESAS-r) questionnaire. Patients with any ESAS-r symptom score $\geq 4$ (moderate-severe) were eligible for analysis. A stratified sampling method was used: 100 patients were randomly selected with 20 patients per year from 2012 to 2016. Patient, tumor and treatment characteristics were extracted from medical records. Health care provider (HCP) documentation of elevated symptoms and subsequent intervention were evaluated. Descriptive statistics were used to report symptom prevalence, HCP documentation and intervention. Fisher's exact test evaluated documentation and intervention rates according to symptom severity and total ESAS-r score.

Results: Between January 2012 and December 2016, 5849 patients completed the ESAS-r. Symptoms scores were $\geq 4$ in 3216 patients (55\%). In our sample of 100 , ovarian (42\%) and endometrial (34\%) malignancies were most common. Median age was 55 years (range 47-63). Median ESAS-r score was 24 (range: 5-84), with tiredness being the most prevalent symptom reported by patients. HCP documented at least one elevated symptom in 50 patients (50\%), most commonly for pain $(71 \%)$ and least commonly for nausea (4\%). Subsequent interventions were offered to only 32 patients $(32 \%)$, most commonly for pain (56\%). Higher median total ESAS-r score was associated with higher rate of documentation $(p=0.004)$ and higher rate of intervention $(\mathrm{p}<0.001)$.

Conclusions: A significant proportion of gynecologic oncology patients report symptom that should prompt an intervention. However, HCPs documented symptoms in only half of patients and reported interventions in only one third. These results highlight gaps and opportunities for improving symptom screening and response in the "real-world" setting.

\section{Summary}

Although patient-reported outcomes have proven benefits to oncology patients, a knowledge gap exists regarding implementation practices in routine clinical care. In this study, we evaluated rates of health-care provider documentation and subsequent intervention to elevated patient-reported symptoms in a cohort of gynecologic oncology patient. More than half of the gynecologic oncology patients reported moderatesevere symptoms. However, only half of health care providers documented patient symptoms and onethird offered a subsequent intervention. These results highlight opportunities for improving symptom screening and response in the "real-world" setting.

\section{Background}


Patient-reported outcomes (PROs) are defined as "any report coming directly from the patient about a health condition and its treatment [1]." Incorporating PROs into oncology practice has been shown to better identify patients' symptoms, facilitate patient-physician communication, promote patient-centered care and more recently, improve overall survival [2-5]. However, the full potential of symptom screening in clinical practice has not been evaluated, leading to a gap in knowledge regarding implementation practices.

In 2007, Cancer Care Ontario (CCO) introduced routine symptom screening assessment for all regional cancer centres using the Edmonton Symptom Assessment System-revised (ESAS-r). The ESAS-r questionnaire screens for patient-reported distress and has been validated in oncology populations [6, 7]. CCO uses symptom screening rates as a quality indicator for regional cancer centre programmatic assessment. Symptom response algorithms have been created for each individual symptom[8]. Therefore, PROs can be used clinically for symptom assessment and intervention and not just for screening. This provides opportunities for teaching patient self-management techniques as well as advising health care professionals on actionable interventions. Evaluation of ESAS-r implementation in Ontario found that its use was endorsed by patients and HCPs, improved team and patient-clinician communication and increased attention to patients' psychosocial needs [9].

Clinical implementation of PROs can only be sustainable if there is adequate engagement from both patients and HCPs. For example, PRO measures need to be completed by patients prior to their clinical encounter, discussed with their HCP and acted upon, when appropriate. According to Li et al., the key factors for successful implementation of symptom screening include: screening for more than emotional distress, initiating clinic-based first responses, supporting ownership by ambulatory oncology clinics, leveraging a top-down and bottom-up approach, and investing in a programmatic approach [9]. One study evaluating HCP documentation and intervention to elevated pain and shortness of breath symptoms scores in a "real-world" setting of breast and lung cancer patients unfortunately reported low documentation and intervention rates overall. However, there was a positive association between symptom severity and HCP response [10].

Although the benefits of PROs have been established in the oncology research setting[11], the extent of PRO implementation and its resultant impact within a clinical setting is currently unknown. Further investigations into the extent of HCP use of PROs would facilitate effective implementation strategies and identify opportunities for improvement. Therefore, the objective of this study was to evaluate HCP documentation to elevated ESAS-r symptom scores and subsequent interventions in a Gynecologic oncology clinic at a large, academic tertiary care cancer centre.

\section{Methods}

\section{Design and setting:}


This was a retrospective chart review of Gynecologic Oncology patients who were seen in routine ambulatory clinics within a single tertiary care cancer centre between January 1, 2012 and December 31, 2016. This timeframe was chosen as the ESAS-r was implemented into Gynecologic oncology clinics during 2011. Within our institution, the Gynecologic Oncology clinic is supported by radiation, medical and gynecologic oncologists and specialized oncology nurses. Gynecologic oncology clinics were chosen because this patient population is known to have a high prevalence of disease-related and treatmentrelated symptoms [12-14]. Institutional research ethics board approval was obtained.

\section{Symptom screening:}

The ESAS-r is a validated PRO tool that assesses nine symptoms commonly experienced by cancer patients (pain, tiredness, nausea, depression, anxiety, drowsiness, appetite, well-being and shortness of breath) on a Likert scale from "0- symptom is absent" to "10-worst possible severity" [15]. Patients complete the ESAS-r on paper or electronically on iPads in the clinic waiting areas prior to their clinical encounter. Symptom response pathways have been created for the ESAS-r. For low distress scores (mild symptoms) corresponding to items rated $1-3$, patients are directed to education resources for selfmanagement of symptoms, whereas moderate-high scores corresponding to items rated $\geq 4$, require further assessment by the primary oncology team or referral to specialized services [16]. A stratified sampling method was used by randomly selecting an equal number of patients from each year between 2012 and 2016 to make up a sample size of 100 charts for chart audit.

\section{Chart Reviews and Coding:}

The ESAS-r data for all patients seen in gynecologic oncology clinics between January 1, 2016 and December 31, 2016 were obtained from our internal Distress Assessment and Response Tool (DART) ecancer database. For this study, only patients who completed the ESAS-r and had a symptom score $\geq 4$ (moderate/severe) were eligible. For the randomly selected 100 patients, the chart review date corresponded to the date of the clinical encounter with an elevated ESAS-r symptom score. Patient, tumor and treatment characteristics were extracted from electronic medical records as well as the type of clinic visit (e.g. new consult, radiation review, follow-up). Charts were reviewed for HCP documentation of ESASr symptom scores $\geq 4$ and subsequent interventions by two independent oncologists. HCP documentation was defined as any written/dictated documentation to any of the 9 ESAS-r symptoms with a score $\geq 4$ on the date of the clinical encounter. HCP intervention was defined as any subsequent action taken by a HCP in response to an elevated symptom score of $\geq 4$ and was defined as patient education, a treatment recommendation (e.g. prescription for analgesics, cancer-directed treatment), an investigation request (e.g. imaging) and a specialist referral (e.g. palliative care)

The following data sources were used: dictated physician notes, electronic notes for nursing and allied health professionals ("Patient Care Notes"), and MOSAIQ notes. Symptom-related interventions were also categorized as follows: patient education (e.g. pamphlets describing self-management of symptoms), treatment recommendation (e.g. prescription for analgesics), investigation (e.g. CT scan) or referral (e.g. 
palliative care, psychosocial). The results were reviewed by the two oncologists for agreement and consensus. In cases of discrepancies the charts were reviewed together and discussed until agreement.

\section{Statistical Analyses:}

Summary statistics were used to describe the patient, disease and treatment characteristics, as well as each ESAS-r component and total score, with frequency and percentage for categorical variables and median and range for continuous variables. Boxplot was used to visualize each ESAS-r component and the symptom-specific HPC documentation/ intervention. Mann-Whitney U test was used to assess the association between each component score and the corresponding documentation/intervention. Univariate and multivariable logistic regression were used to assess factors that may be associated with any documentation and any intervention respectively. All tests are two-sided, and a p-value less than 0.05 is considered as statistically significant.

\section{Results}

Between January 2012 and December 2016, 5849 gynecologic oncology unique patients completed the ESAS-r. At least one item of the ESAS score was $\geq 4$ in 3216 patients (55\%) and $\geq 7$ in 1446 patients $(25 \%)$.

In our sample of 100 unique patients, ovarian (42\%) and endometrial (34\%) malignancies were the most common. Median age was 55 years (range 47-63) and most prevalent age group was 60-74 years old $(57 \%)$. The majority of patients (68\%) were attending a follow-up appointment (Table 1$)$. Overall, the median total ESAS-r score was 24 (range: 5-84). The most common patient-reported symptoms were tiredness $(71 \%)$ and anxiety $(61 \%)$ and the least prevalent patient-reported symptoms were drowsiness (25\%), and nausea (23\%) (Fig. 1). For patients' population who did not meet the ESAS inclusion criteria, the median total ESAS score was 3(range 0-25)

Table 1: Patients Characteristics $(n=100)$ 


\begin{tabular}{ll} 
Characteristic & \multicolumn{1}{c}{$\mathrm{N}[\%]$} \\
\hline Age at diagnosis & \\
Mean (sd) & $54.5(13.6)$ \\
(median (range), [IQR]) & $55.00(19-86),[47.00-63.25]$ \\
\hline Site [\%] & \\
Cervix & $16[16.0]$ \\
Endometrium & $34[34.0]$ \\
Ovary & $42[42.0)$ \\
Vulva & $5[5.0]$ \\
Others & $3[3.0]$ \\
*FIGO & \\
I & $51(15)$ \\
II & $13(13)$ \\
III & $20(20)$ \\
IV & $15(15)$ \\
missing & $1(1)$ \\
Visit type [\%] & \\
Consult & $26[26.0]$ \\
Follow Up & $70[70.0]$ \\
Radiation Review & $4[4.0]$
\end{tabular}

*Fédération Internationale de Gynécologie et d'Obstétrique

In our sample of 100 patients, HCP documentation of at least one elevated symptom (ESAS-r symptom score $\geq 4)$ occurred in 50 patients $(50 \%)$, most commonly for pain (71\%) and least commonly for depression (11\%) and nausea (4\%). HCP intervention was offered to only 32 patients (32\%), most commonly for pain (56\%) and least commonly for depression (8\%) and nausea (4\%) (Fig. 2). The most common intervention was a treatment recommendation (12/32, 38\%), such as an opioid prescription (Table 2).

Table 2: ESAS-r Scores for all patients $(n=100)$ 


\begin{tabular}{|ll|}
\hline Characteristic & $\mathrm{N}[\%]$ \\
\hline ESAS-r Score & \\
Mean (sd) & $28.9[16.8]$ \\
Median ESAS-r score [range] & $24[5-84]$ \\
Median ESAS-r score per visit type [range]: & \\
• Follow up appointments ( $\mathrm{n}=70)$ & $23[5-70]$ \\
• Consult ( $\mathrm{n}=26)$ & $26[9-55]$ \\
& $33.5[13-56]$ \\
\hline Documentation review & \\
No & $50[50]$ \\
Yes & $50[50]$ \\
\hline Intervention for ESAS-r $\geq 4$ & \\
No & $68[68]$ \\
Yes & $32[32]$ \\
\hline Intervention type & \\
Counselling: & $7[22]$ \\
Treatment & $11[34]$ \\
Investigation & $12[38]$ \\
Referral to GP & $4[12]$ \\
Referral to specialist & $722]$ \\
\hline
\end{tabular}

The higher median total ESAS-r score, indicating greater global symptom burden, was associated with higher rate of both documentation $(p=0.002)$ and intervention $(p<0.001)$. For individual symptoms, higher ESAS-r score was associated with significantly more documentation and intervention for both pain and tiredness only $(p<0.05)$ (Fig. 3).

Univariate analysis was used to assess factors that may be associated with documentation and intervention. Age, pathology, FIGO stage (International Federation of Gynecology and Obstetrics cancer staging, treatments (surgery, chemotherapy, radiation therapy), and total ESAS-r score were included in the analysis. Receipt of chemotherapy [Cl 95\% $4.15(1.8,9.57), \mathrm{p}<0.001]$ and higher total ESAS-r score [Cl $95 \% 1.06$ (1.03,1.09), $p<0.001]$ were associated with higher rates of HCP documentation and intervention. On multivariate analysis, receipt of chemotherapy and higher ESAS-r score were still significantly associated with higher rate of documentation interventions $(p<0.05)$ (Table 3$)$. 
Table 3: Factors associated with higher documentation by health care professional on multivariable analysis

\begin{tabular}{|c|c|c|c|c|c|c|}
\hline \multirow[b]{2}{*}{ Covariate } & \multicolumn{3}{|c|}{ Documentation by * $\mathrm{HCP}$} & \multicolumn{3}{|c|}{ Intervention by *HCP } \\
\hline & OR $(95 \% \mathrm{Cl})$ & $\begin{array}{l}p- \\
\text { value }\end{array}$ & $\begin{array}{l}\text { Global p- } \\
\text { value }\end{array}$ & OR $(95 \% \mathrm{Cl})$ & $\begin{array}{l}\mathrm{p}- \\
\text { value }\end{array}$ & $\begin{array}{l}\text { Global } p \\
\text { value }\end{array}$ \\
\hline $\begin{array}{l}\text { Age at } \\
\text { Diagnosis }\end{array}$ & $\begin{array}{l}0.98 \\
(0.95,1.02)\end{array}$ & & 0.31 & $\begin{array}{l}0.99 \\
(0.95,1.03)\end{array}$ & & 0.55 \\
\hline FIGO & & & 0.36 & & & 0.77 \\
\hline I & Reference & & & Reference & & \\
\hline II & $\begin{array}{l}1.89 \\
(0.45,7.99)\end{array}$ & & & $\begin{array}{l}1.08 \\
(0.23,5.12)\end{array}$ & & \\
\hline III & & 0.4 & & 0.67 & & \\
\hline IVA & $(0.13,2.28)$ & 0.41 & & $(0.14,3.28)$ & & \\
\hline & $\begin{array}{l}1.92 \\
(0.41,8.87)\end{array}$ & & & $\begin{array}{l}1.65 \\
(0.36,7.56)\end{array}$ & & \\
\hline Chemotherapy & & & 0.0038 & & & 0.043 \\
\hline No & reference & & & reference & & \\
\hline Yes & $\begin{array}{l}5.06 \\
(1.69,15.15)\end{array}$ & & & $\begin{array}{l}3.51 \\
(1.04,11.87)\end{array}$ & & \\
\hline TOTAL SCORE & $\begin{array}{l}1.04 \\
(1.01,1.08)\end{array}$ & & 0.0075 & $1.06(1.03,1.1)$ & & 0.001 \\
\hline
\end{tabular}

*HCP: Health care professional

\section{Discussion}

This study evaluated HCP documentation of elevated patient-reported symptom scores and subsequent intervention in a sample of patients who were seen at a gynecologic oncology clinic at a large academic cancer centre. Although a significant proportion of patients reported elevated ESAS-r scores of at least one of the nine symptoms, HCPs documented at least one ESAS-r symptom in only half of patients and offered subsequent interventions in only one third.

There is a paucity of literature evaluating PROs in clinical practice, yet our results are consistent with, or slightly higher than, these publications. In our study cohort, tiredness was the most prevalent self-reported symptom by patients, whereas nausea was least prevalent. Similarly, in a study of ambulatory cancer patients in Ontario, Barbera et al., reported fatigue as the most prevalent symptom (75\%) and nausea least prevalent $(25 \%)$. More than half of patients reported pain and shortness of breath. This study encompassed all cancer types along all cancer journey trajectories, with lung cancer patients having the 
highest symptom burden [17]. In a retrospective study that included 391,305 patients diagnosed with cancer between January 2007 and December 2014, pain was found to be highest in lung cancer patients and lowest in gastrointestinal cancer patients [18]. In a retrospective study involving stage I-III breast cancer patients within the first year of diagnosis, tiredness was the most prevalent moderate-to-severe symptom (60\%), whereas nausea was the least prevalent (15\%) [19]. These results underscore the differences in symptom burden that exist between various cancer sites and the importance of understanding these differences when initiating a PRO program within an institution. For example, some cancer sites benefit from symptom monitoring using generic PRO tools, such as the ESAS-r, whereas other sites may benefit more using disease-specific PRO tools.

HCPs documented elevated symptoms in half of patients and offered corresponding interventions in only approximately one-third. A retrospective study evaluating documentation and symptom-specific actions for patient-reported pain and shortness of breath in a cohort of lung and breast cancer patients found that pain was documented in $52 \%$ of clinical charts and shortness of breath in $30 \%$, with subsequent clinical action in $17 \%$ and $4 \%$ respectively [10]. Additionally, a German cancer centre reported their implementation and results of electronic real-time assessment of PROs in routine care and found that although patient acceptability was high, physician uptake was low overall as they had low usage of PROs and reported irrelevance of some of the items and lack of knowledge about other items as potential barriers[20].

The symptoms of fatigue and pain were most commonly documented, whereas nausea and psychosocial symptoms of anxiety and depression were documented least commonly. Furthermore, a correlation was found between rates of documentation and intervention and ESAS-r scores, as well as receipt of chemotherapy. A population-based study evaluating opioid prescription after pain assessment using the ESAS-r found that the proportion of patients with a prescription increased as pain severity increased; however, one-third of patients with severe pain did not have any clinical action[21]. Another controlled intervention study reported that awareness of HCP of collected PROs and subsequent intervention improved patient pain outcome. The study included 264cancer patients with pain score of $\geq$ 4. All patients completed baseline and follow up questionnaires after 2-5 days using Brief Pain Inventory (BPI) and the ESAS questionnaires. The completed questionnaires by the intervention group were documented in the electronic medical record and presented to the HCP who were provided with pain management guideline on request. The HCP did not have access to the control group questionnaires. The study reported statistical significant improvement of symptoms on the follow up questionnaire compared with the baseline in the intervention group for almost all items of PPI and ESAS compared with the controlled group[22]. These results highlight the utility of symptom screening in routine clinical practice. However, the lack of response and intervention remains too high, suggesting opportunities to improve symptom screening and enhance symptom management.

Psychosocial symptoms are less commonly documented and acted upon by HCPs [23, 24]. There are possible explanation for this; first, during cancer treatment there may be a tendency for HCPs to focus on physical symptoms that directly related to disease-burden or treatment toxicity. Additionally, HCPs may 
feel less equipped to manage psychosocial symptoms and may believe other HCPs, such as social work and/or palliative care, are more appropriate. Furthermore, patients might perceive their oncologist expertise as solely treating cancer. CCO has developed symptom management algorithms for all ESAS-r symptoms, including anxiety and depression[25]. These resources could help educate HCPs as they attempt to respond to elevated symptom scores.

Although symptom monitoring has been mandated on a provincial level, a gap remains between desired state and current clinical practice warranting further investigation. Currently, screening rates serve as a quality indicator for cancer centre programmatic performance; however, one could call into question whether this is the most appropriate metric and whether documentation and/or intervention rates should also be considered. Potential barriers to the successful use of PROs in clinical practice include the perception amongst HCPs that PROs increase the length of the clinical encounter, electronic systems are unable to optimally support PRO use and clinical workflow and pressures challenge PRO integration[26]. In order to ensure optimal implementation of PROs within clinical care, workflow processes need to be created and streamlined with clinic workflow. In a study by Berry et al, the use of an automated PRO tool helped flag issues reported by patients for discussion, and did not prolong the clinic visit [27].

We acknowledge the limitations of this study. First, it is a retrospective chart review within a single clinical site at once cancer centre. Secondly, although recommended, not all patients complete the ESAS-r at the time of their clinical encounter and therefore, it is unknown what their symptom burden was. Thirdly, some HCPs may have responded to, or acted upon, elevated symptom scores without documenting these interactions in the clinical chart. We emphasize the importance of documentation to ensure effective communication within and the patient's HCP team and beyond. Lastly, as this is an audit study, the ESAS$r$ score was reviewed at only one time point and therefore, it is unknown whether the previous and subsequent ESAS-r scores would impact our analysis.

\section{Conclusion And Future Directions:}

Although patient-reported screening has been mandated at the provincial level, a significant proportion of gynecologic oncology patients report elevated symptom scores that should prompt an intervention. However, in this study we found that HCPs documented elevated symptoms in only half of patients and offered actionable interventions in only one third. These results highlight the importance of evaluating implementation practices. Opportunities exist for improving symptom screening in the "real-world," perhaps through integration of electronic platforms and nurse-led assessment clinics. It is unlikely that a "one size fits all" approach will be successful. As such, integration of PROs in clinical practice may require customized implementation processes for different clinical sites to ensure successful use, documentation and subsequent documented intervention.

\section{Declarations}

Funding: N/A 


\section{Conflicts of interest/Competing interests:}

Dr. S. L'heureux has honoraria from Roche, Merck, AZ, GSK and Eisai and is the PI or Co-PI of different clinical industry-sponsored or Investigator-initiated trials. All other authors have no conflict of interest.

Availability of data and material: Data available

Code availability: Not applicable

Authors' contributions: All authors reviewed and revised the manuscript accordingly and participated in approval of the final manuscript. Material preparation, data collection and analysis were performed by [Soha Atallah], [Jennifer Croke] and [ZhihuiAmy Liu]. The first draft of the manuscript was written by [Soha Atallah] and all authors commented on previous versions of the manuscript. All authors read and approved the final manuscript.

Ethics approval: Study was approved by Institutional research ethics board

Consent to participate: This is a retrospective study. Patient consent was not required

Consent for publication: All authors consented for publishing the manuscript. No individual data or image were submitted

\section{References}

1. Willke, R.J., L.B. Burke, and P. Erickson, Measuring treatment impact: a review of patient-reported outcomes and other efficacy endpoints in approved product labels. Control Clin Trials, 2004. 25(6): p. 535-52.

2. Kotronoulas G, K.N., Maguire R, Harrow A, Di Domenico D, Croy S, MacGillivray S., What is the value of the routine use of patient-reported outcome measures toward improvement of patient outcomes, processes of care, and health service outcomes in cancer care? A systematic review of controlled trials. J Clin Oncol, 2014. 32(14): p. 1480-501.

3. G Velikova, L.B., AB Smith, PM Brown, Measuring quality of life in routine oncology practice improves communication and patient well-being: a randomized controlled trial. J Clin Oncol, 2004. 22(7): p. 14-24.

4. Ethan Basch, A.M.D., Amylou C. Dueck; et al, Overall Survival Results of a Trial Assessing PatientReported Outcomes for Symptom Monitoring During Routine Cancer Treatment. JAMA, 2017. 318(2): p. 197-198.

5. Basch, E., et al., Symptom Monitoring With Patient-Reported Outcomes During Routine Cancer Treatment: A Randomized Controlled Trial. J Clin Oncol, 2016. 34(6): p. 557-65. 
6. Chang VT, H.S., Feuerman M, Validation of the Edmonton Symptom Assessment Scale. Cancer, 2000. 88(9): p. 2164-71.

7. Watanabe, S.M., C.L. Nekolaichuk, and C. Beaumont, The Edmonton Symptom Assessment System, a proposed tool for distress screening in cancer patients: development and refinement. Psychooncology, 2012. 21(9): p. 977-85.

8. Cancer Care Ontario, S.M.T.

9. Li M, M.A., Crawford S, Bagha S, Leung YW, Zimmermann C, Fitzgerald B, Wyatt M, Stuart-McEwan T, Rodin G., Easier Said Than Done: Keys to Successful Implementation of the Distress Assessment and Response Tool (DART) Program. J Oncol Pract, 2016. 12(5): p. e513-26.

10. Seow, H., et al., Do high symptom scores trigger clinical actions? An audit after implementing electronic symptom screening. Journal of oncology practice, 2012. 8(6): p. e142-e148.

11. Basch, E., et al., Overall survival results of a trial assessing patient-reported outcomes for symptom monitoring during routine cancer treatment. JAMA, 2017. 318(2): p. 197-198.

12. Bjelic-Radisic, V., et al., Quality of life characteristics inpatients with cervical cancer. Eur J Cancer, 2012. 48(16): p. 3009-18.

13. Kathrin Kirchheiner'Correspondence information about the author Kathrin KirchheinerEmail the author Kathrin Kirchheiner, R.A.N., Agnieszka Czajka-Pepl, Elisabeth Ponocny-Seliger, Alina E. Sturdza, Johannes C. Dimopoulos, Wolfgang Dörr, Richard Pötter, Health related quality of life and patient reported symptoms before and during definitive radio(chemo)therapy using image-guided adaptive brachytherapy for locally advanced cervical cancer and early recovery - a mono-institutional prospective study. Gynecol Oncol., 2015. 136(3): p. 415-23.

14. Le Borgne, G., et al., Quality of life in long-term cervical cancer survivors: a population-based study. Gynecol Oncol, 2013. 129(1): p. 222-8.

15. Bruera, E., et al., The Edmonton Symptom Assessment System (ESAS): a simple method for the assessment of palliative care patients. J Palliat Care, 1991. 7(2): p. 6-9.

16. Selby, D., et al., A single set of numerical cutpoints to define moderate and severe symptoms for the Edmonton Symptom Assessment System. J Pain Symptom Manage, 2010. 39(2): p. 241-9.

17. Barbera, L., et al., Symptom burden and performance status in a population-based cohort of ambulatory cancer patients. Cancer, 2010. 116(24): p. 5767-76.

18. Bubis, L.D., et al., Symptom Burden in the First Year After Cancer Diagnosis: An Analysis of PatientReported Outcomes. J Clin Oncol, 2018. 36(11): p. 1103-1111. 
19. Davis, L.E., et al., Patient-reported symptoms after breast cancer diagnosis and treatment: $A$ retrospective cohort study. Eur J Cancer, 2018. 101: p. 1-11.

20. Trautmann, F., et al., Electronic real-time assessment of patient-reported outcomes in routine care-first findings and experiences from the implementation in a comprehensive cancer center. Support Care Cancer, 2016. 24(7): p. 3047-56.

21. Barbera, L., et al., Opioid prescription after pain assessment: a population-based cohort of elderly patients with cancer. J Clin Oncol, 2012. 30(10): p. 1095-9.

22. Andersson, V., et al., Benefits of using the Brief Pain Inventory in patients with cancer pain: an intervention study conducted in Swedish hospitals. Supportive Care in Cancer, 2019.

23. Hardman, A., P. Maguire, and D. Crowther, The recognition of psychiatric morbidity on a medical oncology ward. J Psychosom Res, 1989. 33(2): p. 235-9.

24. Coyne, J.C., et al., Distress, psychiatric morbidity, and prescriptions for psychotropic medication in a breast cancer waiting room sample. Gen Hosp Psychiatry, 2004. 26(2): p. 121-8.

25. Howell D, K.H., Esplen MJ, Hack T, Hamel M, Howes J, Jones J,Li M,Manii D,McLeod D, Mayer C, Sellick S, Riahizadeh S, Noroozi H, \&Ali M, on behalf of the Cancer Journey Advisory Group of the Canadian Partnership Against Cancer. A Pan Canadian Practice Guideline: Screening, Assessment and Care of Psychosocial Distress, Depression, and Anxietyin Adults with Cancer, Toronto: Canadian Partnership Against Cancer and the Canadian Association of Psychosocial Oncolog. 2015(July).

26. Anatchkova, M., et al., Exploring the implementation of patient-reported outcome measures in cancer care: need for more real-world evidence results in the peer reviewed literature. J Patient Rep Outcomes, 2018. 2(1): p. 64.

27. Berry, D.L., et al., Enhancing patient-provider communication with the electronic self-report assessment for cancer: a randomized trial. J Clin Oncol, 2011. 29(8): p. 1029-35.

\section{Figures}




\section{ESAS-r $\geq 4$}

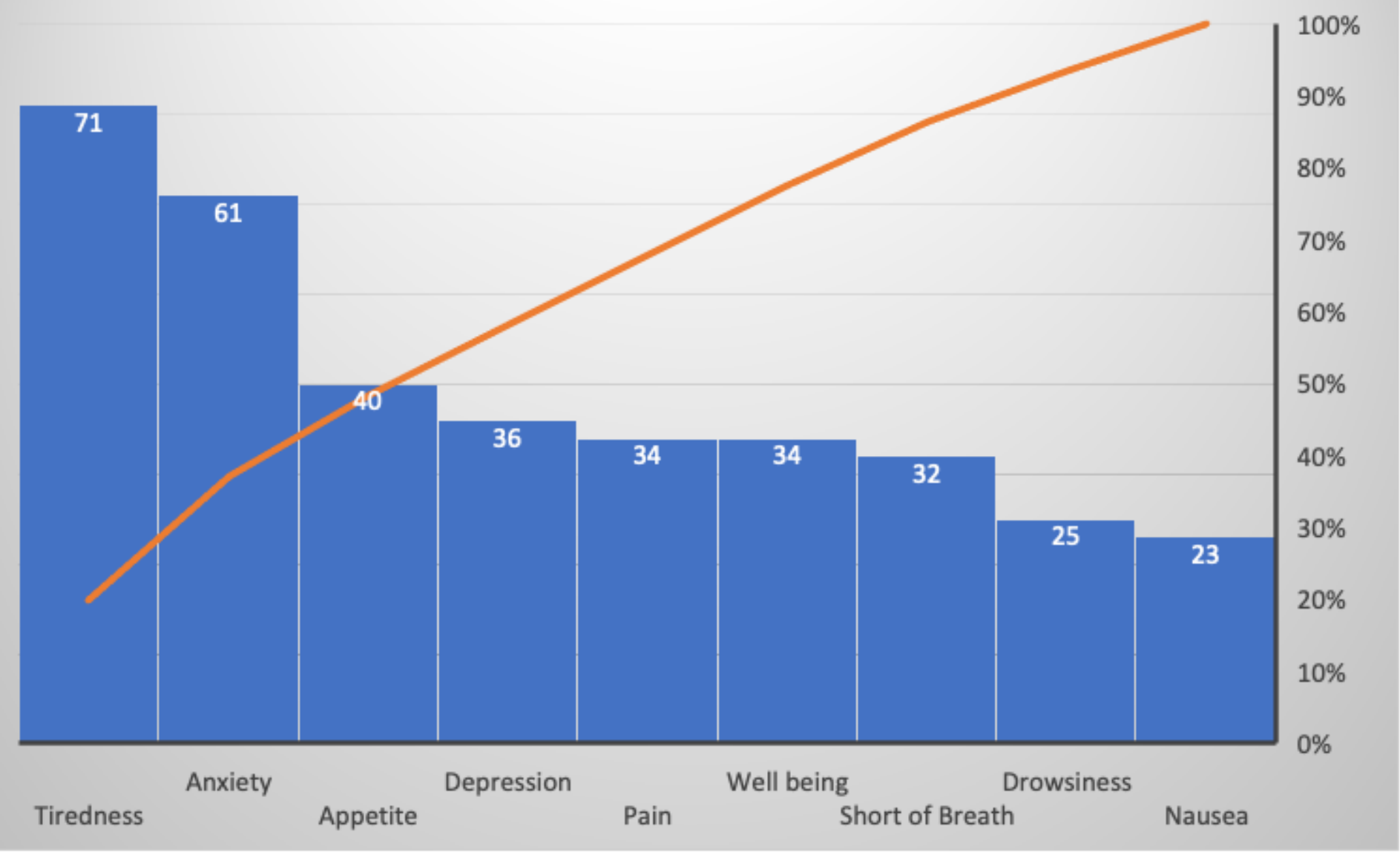

\section{Figure 1}

Distribution of $\geq 4$ ESAS-r symptoms reported by patients 


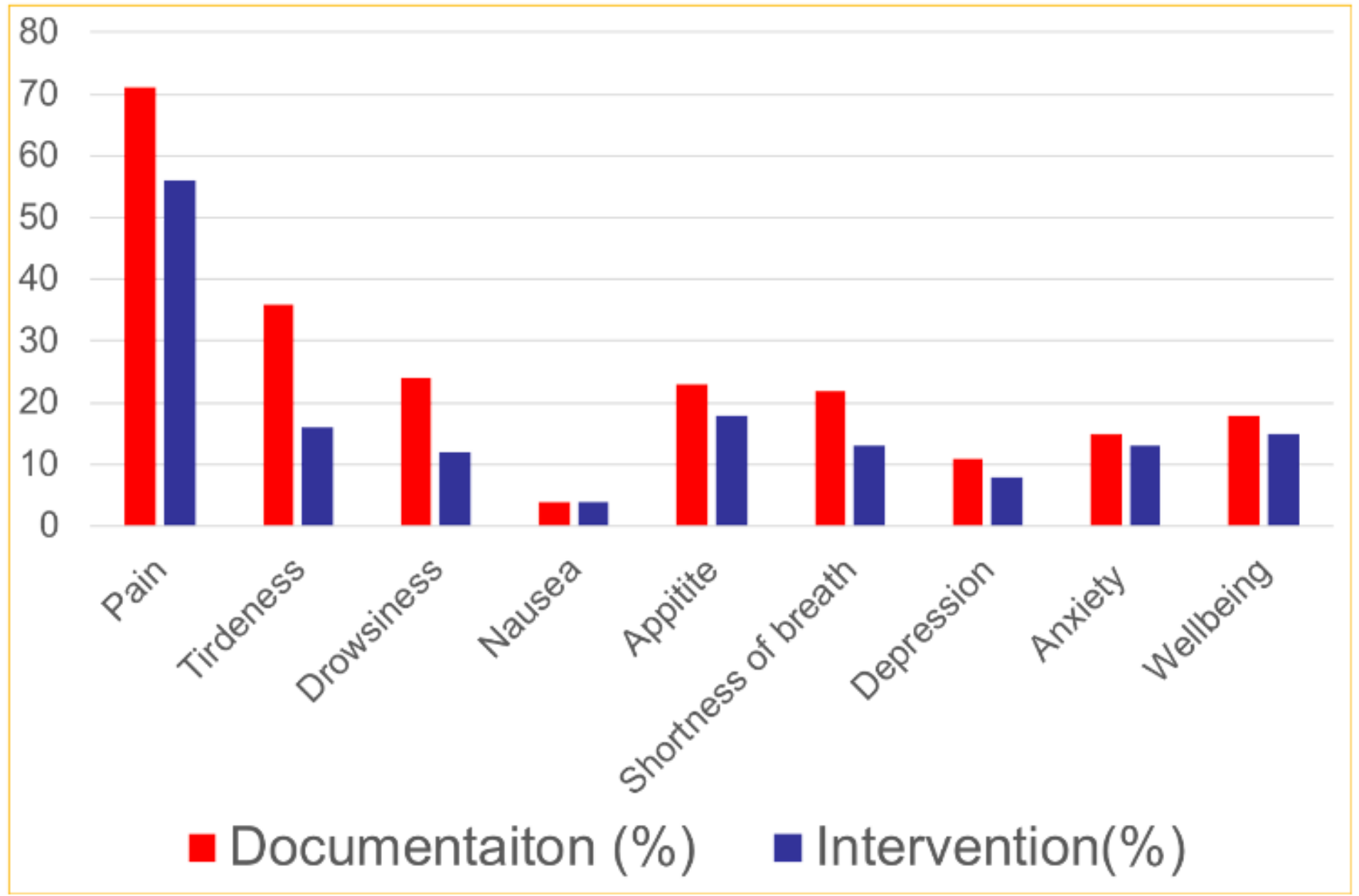

Figure 2

Frequency of documentation and Intervention for ESAS-r symptoms 

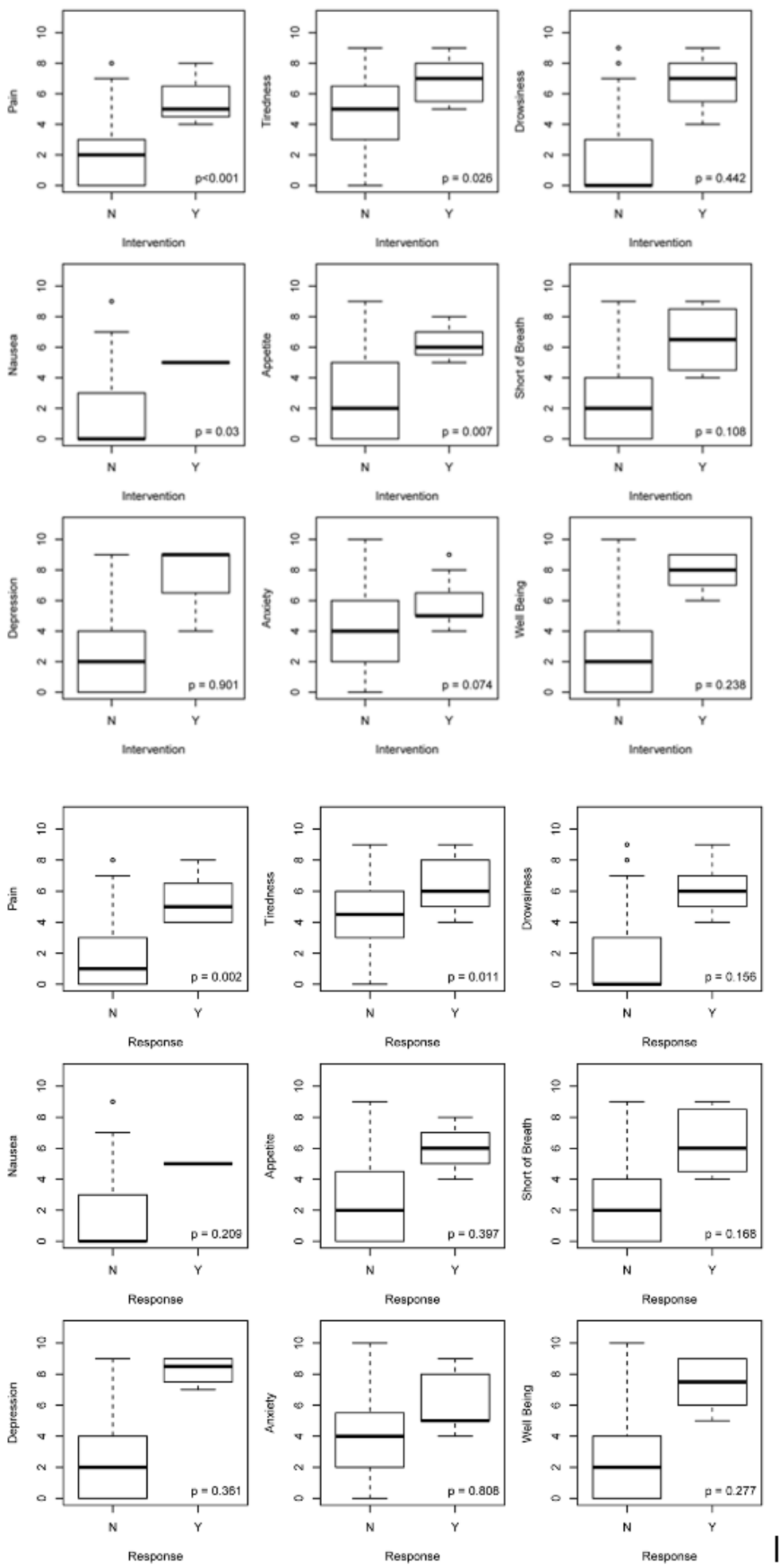

Figure 3

Association between ESAS score and documentation by health care professional 